\title{
Radio observations of the region around the pulsar wind nebula HESS J1303-631 with ATCA
}

\author{
Iurii Sushch $^{1,2,3}$, Igor Oya ${ }^{2}$, Ullrich Schwanke ${ }^{4}$, Simon Johnston ${ }^{5}$, and Matthew L. Dalton ${ }^{6}$ \\ 1 Centre for Space Research, North-West University, 11 Hoffman Street, Potchefstroom 2520, South Africa \\ e-mail: iurii.sushch@nwu.ac.za \\ 2 DESY, Platanenallee 6, 15738 Zeuthen, Germany \\ e-mail: [iurii.sushch;igor.oya.vallejo]@desy.de \\ 3 Astronomical Observatory of Ivan Franko National University of L'viv, vul. Kyryla i Methodia, 8, L'viv 79005, Ukraine \\ 4 Institut für Physik, Humboldt-Universität zu Berlin, Newtonstr. 15, 12489 Berlin, Germany \\ 5 Australia Telescope National Facility, CSIRO, PO Box 76, Epping, NSW 1710, Australia \\ 6 Active Space Technologies GmbH, Carl Scheele Strasse 14, 12489 Berlin, Germany
}

Received 1 December 2015 / Accepted 17 June 2017

\begin{abstract}
Radio observations of the region surrounding PSR J1301-6305 at 5.5 GHz and 7.5 GHz were conducted with ATCA on September 5, 2013. The observations were dedicated to the search of the radio counterpart of the evolved pulsar wind nebula (PWN) HESS J1303-631, which has been detected in X-rays and GeV-TeV $\gamma$-rays. The collected data do not reveal any significant extended emission associated with PSR J1301-6305. In addition, archival $1.384 \mathrm{GHz}$ and $2.368 \mathrm{GHz}$ data do not show any evidence for a radio counterpart of HESS J1303-631. Archival $1.384 \mathrm{GHz}$ observations reveal the detection of an extended structure centred at an angular distance of $19^{\prime}$ from the pulsar. This extended structure might be a supernova remnant (SNR) and a potential birth place of PSR J1301-6305. The implications of the lack of a radio counterpart of HESS J1303-631 on the understanding of the nature of the PWN are discussed.
\end{abstract}

Key words. radiation mechanisms: non-thermal - radio continuum: stars - pulsars: individual: PSR J1301-6305 stars: winds, outflows

\section{Introduction}

HESS J1303-631 is one of the most prominent examples of the so-called very high energy (VHE; $E>100 \mathrm{GeV}$ ) $\gamma$-ray dark sources, which were detected in the VHE band but do not have counterparts at other energy bands. HESS J1303-631 was discovered in 2005 (Aharonian et al. 2005) but the nature of this source was unclear until 2012, when a detailed study of the energy-dependent morphology provided evidence of its association with the pulsar PSR J1301-6305 (Abramowski et al. 2012). With the increase of the energy threshold, a very extended emission region $\left(\sim 0.4^{\circ} \times 0.3^{\circ}\right.$ at the [0.84-2] $\mathrm{TeV}$ band $)$ of $\mathrm{VHE}$ $\gamma$-rays shrinks towards the position of the pulsar at $E>10 \mathrm{TeV}$. While at lower energies the peak position of the extended emission region is significantly offset from the position of the pulsar, at energies above $10 \mathrm{TeV}$ the pulsar is coincident with the peak of the $\gamma$-ray emission region (Abramowski et al. 2012). Such an energy-dependent morphology is expected for ancient pulsar wind nebulae (PWNe; Abramowski et al. 2012, and references therein). Young electrons located close to the pulsar are not cooled yet and, thus, very energetic. These energetic electrons generate the VHE emission around the pulsar via inverse Compton (IC) scattering on the cosmic microwave background (CMB) photons. Older, cooled down, lower energy electrons might be spread farther away from the pulsar for several reasons (e.g. proper motion of the pulsar that causes older particles to be left behind and/or particle diffusion), but these energetic electrons can still produce $\gamma$-rays via IC scattering, however at lower energies than the young electrons.
The association of HESS J1303-631 with the pulsar is further supported by the detection of its X-ray counterpart with XMM-Newton (Abramowski et al. 2012). The size of the X-ray PWN is much smaller than the size of the VHE source, extending $2^{\prime}-3^{\prime}$ from the pulsar position towards the centre of the VHE $\gamma$-ray emission region. The much smaller size of the X-ray emitting region can be explained by an effective synchrotron cooling of older electrons to energies too low to generate synchrotron emission in the X-ray energy range and/or owing to the decreasing magnetic field strength in the PWN with time (see e.g. de Jager et al. 2009; Vorster et al. 2013b). The tail-like extension of the X-ray source might be an indication of the proper motion direction of the pulsar triggering speculations about its possible birth place (Abramowski et al. 2012).

An analysis of archival data from the Parkes-MIT-NRAO (PMN) survey at $4.85 \mathrm{GHz}$ (Condon et al. 1993) also revealed a hint of radio emission at the pulsar position with a size comparable to the X-ray emission region (Abramowski et al. 2012). Data analysis showed a $\sim 3 \sigma$ feature with a peak flux density of $30 \mathrm{mJy} / \mathrm{beam}$, which is at the detection limit of the survey and was considered as an upper limit. This hint of a radio counterpart of HESS J1303-631 triggered new dedicated observations with the Australian Telescope Compact Array (ATCA), which were conducted in September 2013. Results of these observations are presented in this paper.

Recently, the counterpart of HESS J1303-631 was finally detected at GeV energies with Fermi-LAT (Acero et al. 2013). The morphology of the source is consistent with a Gaussian of width $0.45^{\circ}$. The source is contaminated by the emission of 
Table 1. Details of the ATCA data of the HESS J1303-631 region analysed in this paper.

\begin{tabular}{lllllllll}
\hline \hline Date & Right ascention & Declination & $\begin{array}{l}\text { Time } \\
{[\mathrm{min}]}\end{array}$ & Array & $\begin{array}{l}\text { Frequencies } \\
{[\mathrm{MHz}]}\end{array}$ & $\begin{array}{l}\text { Bandwidth } \\
{[\mathrm{MHz}]}\end{array}$ & $\begin{array}{l}\text { Primary } \\
\text { calibrator }\end{array}$ & $\begin{array}{l}\text { Secondary } \\
\text { calibrator }\end{array}$ \\
\hline 2013-Sep.-05 & $13^{\mathrm{h}} 2^{\mathrm{m}} 10.00^{\mathrm{s}}$ & $-63^{\circ} 5^{\prime} 34.8^{\prime \prime}$ & 656.5 & $1.5 \mathrm{~A}$ & 5500,7500 & 2048 & $1934-638$ & $1352-63$ \\
2006-Oct.-25 & $13^{\mathrm{h}} 3^{\mathrm{m}} 0.400^{\mathrm{s}}$ & $-63^{\circ} 11^{\prime} 11.55^{\prime \prime}$ & 433.8 & EW352 & 1384,2368 & 128 & $1934-638$ & $1421-490$ \\
2007-Mar.-13 & $13^{\mathrm{h}} 3^{\mathrm{m}} 0.400^{\mathrm{s}}$ & $-63^{\circ} 11^{\prime} 11.55^{\prime \prime}$ & 618.1 & $750 \mathrm{D}$ & 1384,2368 & 128 & $1934-638$ & $1329-665$ \\
2007-Apr.-24 & $13^{\mathrm{h}} 3^{\mathrm{m}} 0.400^{\mathrm{s}}$ & $-63^{\circ} 11^{\prime} 11.55^{\prime \prime}$ & 651.8 & $1.5 \mathrm{C}$ & 1384,2368 & 128 & $1934-638$ & $1329-665$ \\
\hline
\end{tabular}

the nearby supernova remnant (SNR) Kes 17 (G304.6 + 0.1), but it is clearly seen above $31 \mathrm{GeV}$. The emission region of the GeV counterpart of HESS J1303-631 is, as expected, larger than the $\mathrm{TeV}$ source, but the morphology of the emission region is very similar and features an extension in the same direction as the TeV source. However, the size of the $\mathrm{GeV}$ source might be slightly overestimated because of the contamination from Kes 17 . Kes 17 is the closest known SNR to the pulsar PSR J1301-6305 located at the angular distance of 37' (Wu et al. 2011; Gelfand et al. 2013). Assuming a distance to the pulsar of $6.6 \mathrm{kpc}$ (Abramowski et al. 2012), this corresponds to the projected distance between the pulsar and the SNR of $71 \mathrm{pc}$. This large distance makes the association of the SNR with the pulsar very unlikely as it would require an unrealistically high pulsar velocity of $\sim 6000 \mathrm{~km} \mathrm{~s}^{-1}$ for the characteristic age of the pulsar of $11 \mathrm{kyr}$ (Manchester et al. 2005).

\section{Observations}

The ATCA observations of the region surrounding PSR J1301-6305 were conducted on September 5, 2013. Observations were performed using the CABB receiver with the $1.5 \mathrm{~A}$ configuration of the array (minimum and maximum baselines of $153 \mathrm{~m}$ and $3000 \mathrm{~m}$, respectively) at 5.5 and $7.5 \mathrm{GHz}$ frequencies and centred at $\alpha=13^{\mathrm{h}} 02^{\mathrm{m}} 10.00^{\mathrm{s}}, \delta=-63^{\circ} 05^{\prime} 34.8^{\prime \prime}$ (J2000.0), at the angular distance of about $3^{\prime}$ from the pulsar position. The array configuration was chosen to match the resolution of the XMM-Newton observations of $\sim 4^{\prime \prime}$, while at the same time to remain sensitive to structures comparable to the size of the X-ray PWN of $\sim 2^{\prime}$. However, the maximum angular scales to which the observations are sensitive are slightly smaller than the size of the X-ray PWN, namely $\sim 1.7^{\prime}$ at $5.5 \mathrm{GHz}$ and $\sim 1.3^{\prime}$ at $7.5 \mathrm{GHz}$, respectively.

The observations were carried out in two modes: CFB $1 \mathrm{M}$ (no zooms), a bandwidth of $2 \mathrm{GHz}$ with $20481-\mathrm{MHz}$ channels in each intermediate frequency (IF) band; and pulsar binning, the same but with the addition of pulsar binning according to the provided ephemerids. The on-source scan time was $656.5 \mathrm{~min}$. Pulsar binning mode was used in observations in order to be able to correctly subtract the pulsar contribution to the total emission to determine the intrinsic emission from HESS J1303-631. However, since no significant emission corresponding to HESSJ1303-631 was detected (see Sect. 3), the subtraction of the data taken in pulsar binning mode was not performed and thus these data were not used in this study. Primary (flux density) and secondary (phase) calibrators were J1934-638 and J1352-63, respectively. The flux density of J1934-638 is $5.00 \mathrm{Jy}$ at $5.5 \mathrm{GHz}$ and $2.97 \mathrm{Jy}$ at $7.5 \mathrm{GHz}$. The phase calibrator was observed every $\sim 30 \mathrm{~min}$. The observation recorded all four linear polarization modes. Details of the collected data are listed in Table 1.

In this paper we also considered archival ATCA data obtained during observations of HESS J1303-631 centred at $\alpha=$ $13^{\mathrm{h}} 03^{\mathrm{m}} 0.400^{\mathrm{s}}, \delta=-63^{\circ} 11^{\prime} 11.55^{\prime \prime}$ (the position of the peak VHE $\gamma$-ray emission), and performed in the $1.384 \mathrm{GHz}$ and
$2.368 \mathrm{GHz}$ bands. The archival data used in the analysis, taken as part of the Reinfrank et al. project C1557, are presented in Table 1. Only the archival data taken with all six antennas and with observational time longer than 100 min were used in the analysis. The source J1934-638 was used as a primary calibrator with flux densities of $14.95 \mathrm{Jy}$ at $1.384 \mathrm{GHz}$ and $11.59 \mathrm{Jy}$ at $2.368 \mathrm{GHz}$. The sources J1421-490 and J1329-665 were used for phase calibration. The maximum angular scale to which observations are sensitive is $\sim 18^{\prime}$ at $1.384 \mathrm{GHz}$ and $\sim 11^{\prime}$ at $2.368 \mathrm{GHz}$.

\section{Data analysis and results}

The data reduction and image analysis was performed using the miriad (Sault et al. 1995) and karma (Gooch 1995) packages. The resulting clean primary beam corrected (restricted to the area of primary beam response above $30 \%$, which corresponds to the radial distance of $5.8^{\prime}$ ) image (Stokes $I$ ) at $5.5 \mathrm{GHz}$ is shown in Fig. 1. The pulsar PSR J1301-6305 is detected at the position $\alpha=13^{\mathrm{h}} 01^{\mathrm{m}} 45.678^{\mathrm{s}} \pm 0.013^{\mathrm{s}}, \delta=-63^{\circ} 05^{\prime} 34.85^{\prime \prime} \pm 0.20^{\prime \prime}$. No significant extended emission coincident with the pulsar position was detected. The fitted image root mean square (rms) noise is calculated using the imsad task at the level of $0.011 \mathrm{mJy} / \mathrm{beam}$. The synthesised beam is an ellipse with the major and minor axes of $3.79^{\prime \prime}$ and $3.65^{\prime \prime}$, respectively, and the positional angle of $-7.6^{\circ}$.

There is no extended emission coincident with the pulsar position detected at $7.5 \mathrm{GHz}$ as well. The fitted image rms noise, calculated using the imsad task, is at the level of $0.011 \mathrm{mJy} / \mathrm{beam}$. The major and minor axes of the beam are $3.06^{\prime \prime}$ and $2.90^{\prime \prime}$, respectively, and the positional angle is $-6.2^{\circ}$.

The analysis of the archival data at $1.384 \mathrm{GHz}$ and $2.368 \mathrm{GHz}$, which combine all the observations listed in Table 1, does not reveal any significant emission coincident with the pulsar either. The observations at $1.384 \mathrm{GHz}$, however, reveal a detection of a shell-like structure to the east of the pulsar position that might potentially be an SNR (see discussion in Sect. 4.1). Figure 2 shows the cleaned and primary beam corrected (restricted to the area of primary beam response above $20 \%$, which corresponds to the radial distance of $25.3^{\prime}$ ) image at $1.384 \mathrm{GHz}$. The SNR candidate G304.4-0.2 is positioned within the black circle. The centre of the structure is at $\alpha=13^{\mathrm{h}} 04^{\mathrm{m}} 31.1^{\mathrm{s}}$, $\delta=-63^{\circ} 02^{\prime} 08^{\prime \prime}$. The fitted image rms noise is estimated to be $0.697 \mathrm{mJy} /$ beam. The major and minor axes of the beam are 51.3" and 43.8", respectively, and the positional angle is $20.9^{\circ}$. The brightest parts of the SNR candidate reach the significance of $13 \sigma$. It is difficult to draw any conclusions about a possible emission from the SNR candidate at $2.368 \mathrm{GHz}$, as these observations are less sensitive to large scale structures, and only a fraction of the SNR candidate is located within the primary beam and the image is distorted by artefacts produced by the strong source MGPS J130237-625718.

Most of the sources detected in the field at $1.384 \mathrm{GHz}$ (Fig. 2), both compact and extended, have counterparts at 


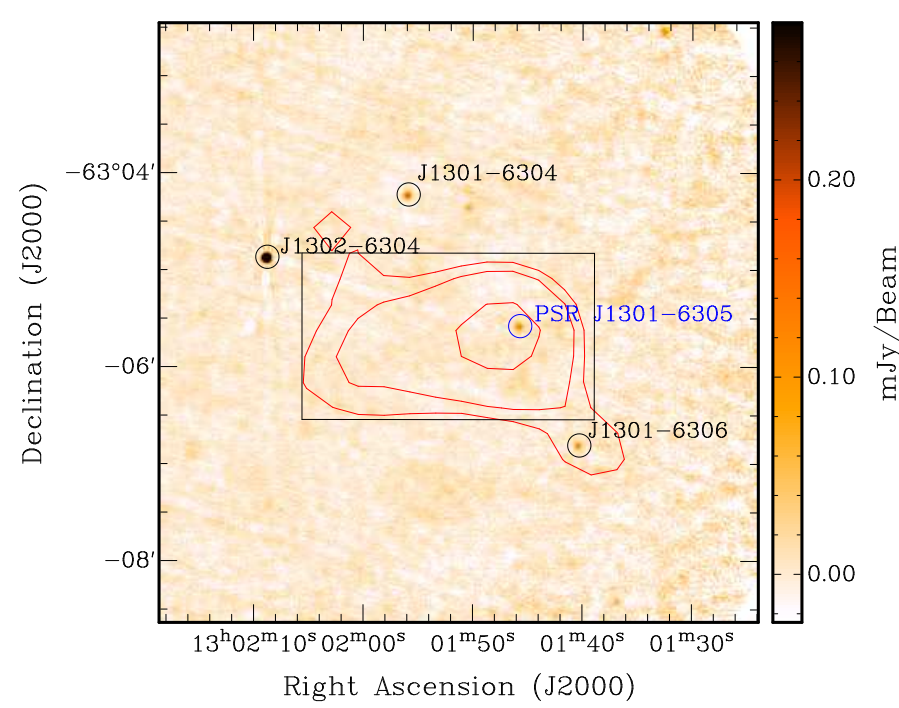

Fig. 1. Radio map of the HESS J1303-631 region at 5.5 GHz overlaid with contours of the X-ray PWN (red) as detected with XMM-Newton (Abramowski et al. 2012). X-ray contours show the peak of the emission close to the pulsar with a trail expanding in the eastern direction The blue circle indicates the position of the pulsar PSR J1301-6305. The black box determines the region used for the flux density upper limit calculation. New point-like sources are indicated with index numbers. The synthesised beam is determined by an ellipse with the major and minor axes of $3.79^{\prime \prime}$ and $3.65^{\prime \prime}$, respectively, and the positional angle of $-7.6^{\circ}$.

other radio frequencies (Murphy et al. 2007; Condon et al. 1993; Schinzel et al. 2015). However, most of these sources are not classified, except for MGPS J130422-624859 (G304.5-0.1), which is identified as a HII region (Misanovic et al. 2002) and MGPS J130037-625324, which is coincident with an infrared bubble (Simpson et al. 2012). Extended emission at the southwestern edge of the field is most probably related to the very extended source PMN J1259-6337 (Wright et al. 1994).

Observations at $1.384 \mathrm{GHz}$ and $2.368 \mathrm{GHz}$ might shed some light on the nature of the unidentified source MGPS J130237-625718, which is also visible at other radio wavelengths (e.g. it is detected in the PMN survey). Both $1.384 \mathrm{GHz}$ and $2.368 \mathrm{GHz}$ data reveal a complex morphology with a central source and two lobes, strongly suggesting that MGPS J130237-625718 is an active galactic nucleus (AGN). Figure 3 shows the map at $2.368 \mathrm{GHz}$ overlaid with contours indicating significant emission from the source at $1.384 \mathrm{GHz}$. Only the observations with the best angular resolution (2007Apr.-24) were used in this analysis. The fitted image rms noise is $0.448 \mathrm{mJy} /$ beam at $1.384 \mathrm{GHz}$ (beam size: major and minor axes of $10.98^{\prime \prime}$ and $9.78^{\prime \prime}$, respectively, with the positional angle of $1.7^{\circ}$ ) and $0.322 \mathrm{mJy} /$ beam at $2.368 \mathrm{GHz}$ (beam size: major and minor axes of 7.15" and 6.65", respectively, with the positional angle of $3.7^{\circ}$ ). A fit to the observed emission at $1.384 \mathrm{GHz}$ and $2.368 \mathrm{GHz}$ was carried out using the imfit task with three Gaussian components. The integrated flux density from the central component is $136.8 \pm 5.0 \mathrm{mJy}$ and $80.8 \pm 3.7 \mathrm{mJy}$ at $1.384 \mathrm{GHz}$ and $2.368 \mathrm{GHz}$, respectively. The flux densities from the southern and northern components are $260.1 \pm 7.3 \mathrm{mJy}$ and $192.5 \pm 6.1 \mathrm{mJy}$ at $1.384 \mathrm{GHz}$ and $156.1 \pm 7.4 \mathrm{mJy}$ and $99.9 \pm 5.5 \mathrm{mJy}$ at $2.368 \mathrm{GHz}$. Unfortunately, the source is outside the primary beam for observations at $5.5 \mathrm{GHz}$ and $7.5 \mathrm{GHz}$ with much better angular resolution.
In the ATCA data presented here, five new point-like sources were detected in the region of HESS J1303-631 (Figs. 1 and 2). Their locations and flux densities estimated using the imsad task are collected in Table 2. Only sources with significance above $10 \sigma$ at both $5.5 \mathrm{GHz}$ and at $1.384 \mathrm{GHz}$ were considered. Each of these sources has one or more potential counterparts in infrared and/or optical catalogues (see e.g. Cutri et al. 2003; Wright et al. 2010; Benjamin et al. 2003; Monet et al. 2003). Two of these sources, J1301-6306 and J1300-6311, have X-ray counterparts in the XMM-Newton catalogue (Rosen et al. 2016). The source J1304-6258 is actually visible in the MGPS-2 (see Fig. 4 right) but is not listed in the catalogue; this is probably because it is very difficult to separate its emission from the extended emission coincident with G304.4-0.2.

\section{Discussion}

The new radio observations reported in this paper were triggered by a hint of a signal $(\sim 3 \sigma)$ from a feature coincident with the X-ray PWN in the analysis of archival data from the PMN survey at $4.85 \mathrm{GHz}$ (Abramowski et al. 2012). This feature is compatible with the resolution of the survey of $\sim 5^{\prime}$ (Condon et al. 1993). These deeper observations at $5.5 \mathrm{GHz}$ and $7.5 \mathrm{GHz}$ with ATCA were optimised for the detection of the putative radio PWN with a size comparable to the size of the X-ray PWN $\left(\sim 2^{\prime}\right)$, while providing a resolution that is comparable to that of XMM-Newton. The assumption of the size was motivated by the size of the possible radio feature and by the hypothesis that the sizes of both radio and X-ray PWNe are constrained by the region of high magnetic field around the pulsar. Indeed, threedimensional magnetohydrodynamic simulations of the Crab nebula (Porth et al. 2014) show that the magnetic field strength close to the termination shock is an order of magnitude higher than the average magnetic field strength in the rest of the PWN. Moreover, in the left-behind relic nebula the magnetic field is expected to be relaxed with the magnetic field strength comparable to the interstellar medium (ISM) magnetic field of about $3 \mu \mathrm{G}$. However, neither new observations at 5.5/7.5 GHz nor the analysis of the archival data at $1.384 / 2.368 \mathrm{GHz}$ show any evidence of extended emission coincident with PSR J1301-6305 and/or the X-ray PWN. It should be noted that the largest scales to which observations at $5.5 / 7.5 \mathrm{GHz}$ are sensitive are slightly smaller than the size of X-ray PWN, $1.7^{\prime}$ at $5.5 \mathrm{GHz}$ and $1.3^{\prime}$ at $7.5 \mathrm{GHz}$, and therefore we cannot rule out the detectability of the putative radio PWN of the size of the X-ray PWN at these frequencies. However, observations at $1.384 / 2.368 \mathrm{GHz}$ allow us to detect structures with an extension up to $\sim 18^{\prime}(1.384 \mathrm{GHz})$ and $\sim 11^{\prime}(2.368 \mathrm{GHz})$. Assuming the size of the X-ray PWN as reported in Abramowski et al. (2012), the upper limits on the radio flux density at $1.384 \mathrm{GHz}$ was estimated at the level of three times the rms noise in the region of the X-ray PWN defined by a box (black box in Figs. 1 and 2). The upper limit on the flux density at $1.384 \mathrm{GHz}$ is $2.6 \mathrm{mJy}$.

However, the size of the putative radio PWN might exceed the size of the largest angular structure resolved in the observations or even the size of the primary beam. GeV observations (Acero et al. 2013) exhibiting a large size of the PWN $\left(\sim 0.9^{\circ}\right)$ indicate that a large amount of relatively low energy relativistic electrons is spread out to large distances from the pulsar. If the size of the putative radio PWN is constrained by the existence of relativistic electrons, i.e. the magnetic field is strong enough for efficient synchrotron emission across the whole PWN, the radio PWN would be at least as large as the 


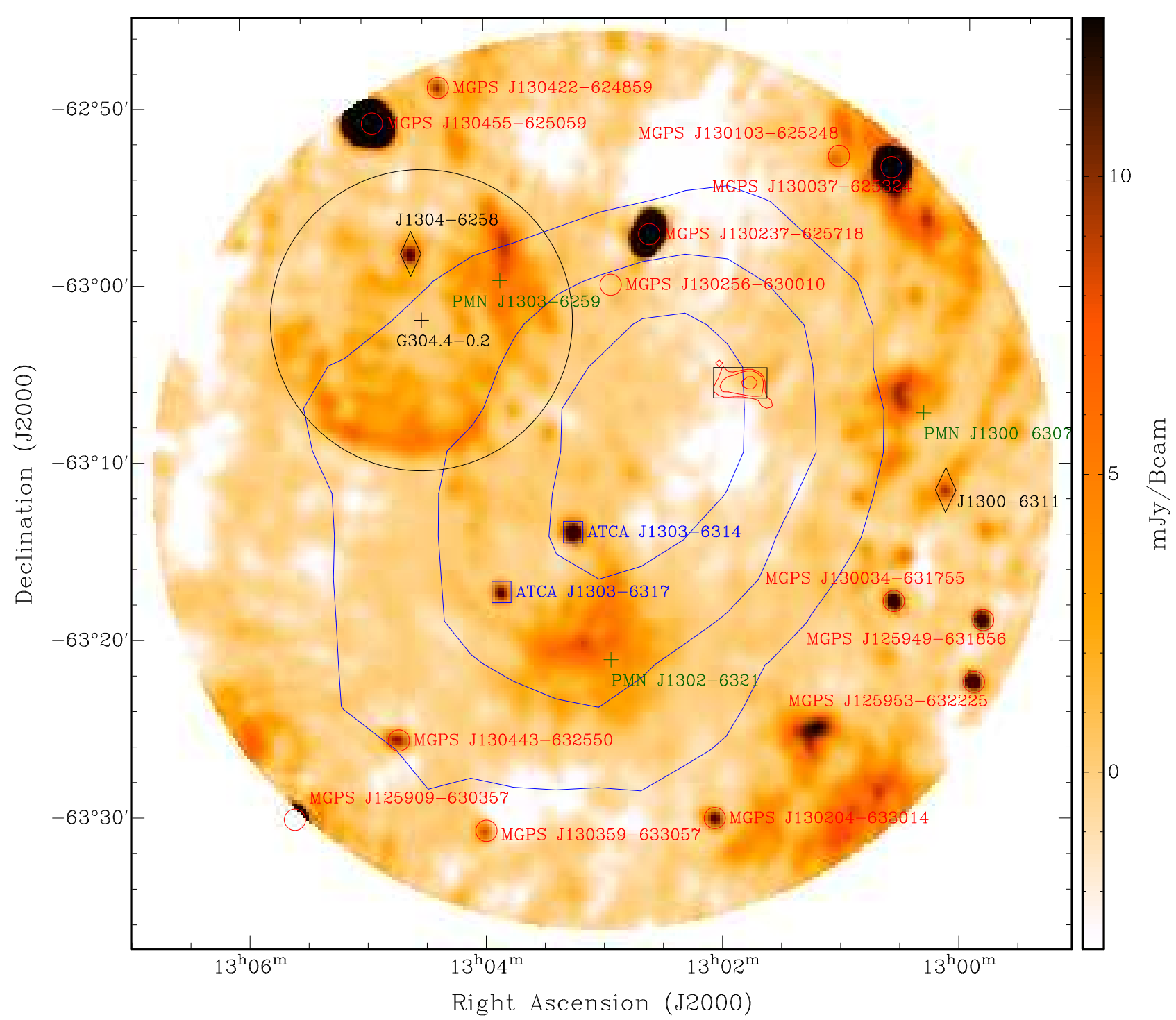

Fig. 2. Radio map of the HESS J1303-631 region at $1.384 \mathrm{GHz}$. Red contours represent the X-ray PWN as detected with XMM-Newton and blue contours represent HESS J1303-631 as detected by H.E.S.S. (Abramowski et al. 2012). The black circle indicates the SNR candidate G304.4-0.2 and the black cross indicates its centre. MGPS-2 catalogue sources (Murphy et al. 2007) are shown as red circles; PMN survey sources (Condon et al. 1993), excluding those which are coincident with MGPS-2 sources, are shown as green crosses and two ATCA sources (Schinzel et al. 2015) are shown as blue squares. The two new point-like radio sources reported in this paper are indicated with black diamonds. The synthesised beam is determined by an ellipse with the major and minor axes of 51.3" and $43.8^{\prime \prime}$, respectively, and the positional angle of $20.9^{\circ}$.

Table 2. New point-like radio sources detected in these observations.

\begin{tabular}{|c|c|c|c|c|c|c|c|}
\hline Identifier & Right ascention & Declination & $\begin{array}{l}F_{5.5 \mathrm{GHz}} \\
{[\mu \mathrm{Jy}]}\end{array}$ & $\begin{array}{l}F_{7.5 \mathrm{GHz}} \\
{[\mu \mathrm{Jy}]}\end{array}$ & $\begin{array}{l}F_{1.384 \mathrm{GHz}} \\
{[\mathrm{mJy}]}\end{array}$ & $\begin{array}{l}F_{2.368 \mathrm{GHz}} \\
{[\mathrm{mJy}]}\end{array}$ & X-ray counterparts ${ }^{a}$ \\
\hline J1301-6306 & $13^{\mathrm{h}} 01^{\mathrm{m}} 40.27^{\mathrm{s}}$ & $-63^{\circ} 06^{\prime} 48.6^{\prime \prime}$ & 127.2 & 118.3 & $\ldots$ & 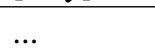 & $38.2-630654^{b}$ \\
\hline J1302-6304 & $13^{\mathrm{h}} 02^{\mathrm{m}} 8.74^{\mathrm{s}}$ & $-63^{\circ} 04^{\prime} 52.1^{\prime \prime}$ & 1448.0 & 1172.0 & $\cdots$ & & none \\
\hline J1301-6304 & $13^{\mathrm{h}} 01^{\mathrm{m}} 55.86^{\mathrm{s}}$ & $-63^{\circ} 04^{\prime} 13.5^{\prime \prime}$ & 191.2 & 119.1 & $\ldots$ & $\cdots$ & none \\
\hline $\mathrm{J} 1300-6311$ & $13^{\mathrm{h}} 0^{\mathrm{m}} 9.23^{\mathrm{s}}$ & $-63^{\circ} 11^{\prime} 37.8^{\prime \prime}$ & $\ldots$ & $\ldots$ & 11.7 & & 3XMM J130006.2-631207 \\
\hline J1304-6258 & $13^{\mathrm{h}} 4^{\mathrm{m}} 36.23^{\mathrm{s}}$ & $-62^{\circ} 58^{\prime} 22.7^{\prime \prime}$ & $\ldots$ & $\ldots$ & 11.3 & 5.7 & none \\
\hline
\end{tabular}

Notes. ${ }^{(a)}$ There are also multiple infrared and/or optical sources that are consistent with the position of these radio sources. ${ }^{(b)}$ Rosen et al. $(2016)$.

$\mathrm{GeV}$ PWN. The same electrons that emit GeV $\gamma$-rays via inverse Compton scattering on ambient photon fields are also responsible for the generation of the radio emission via the synchrotron mechanism (see e.g. Vorster et al. 2013b, and references therein).

\subsection{G304.4-0.2: an SNR or not}

The extended structure, G304.4-0.2, detected to the east of the pulsar at $1.384 \mathrm{GHz}$ exhibits a shell-like morphology. Such a morphology naturally suggests that this might be an SNR. G304.4-0.2 is coincident with PMN J1303-6259, an extended 


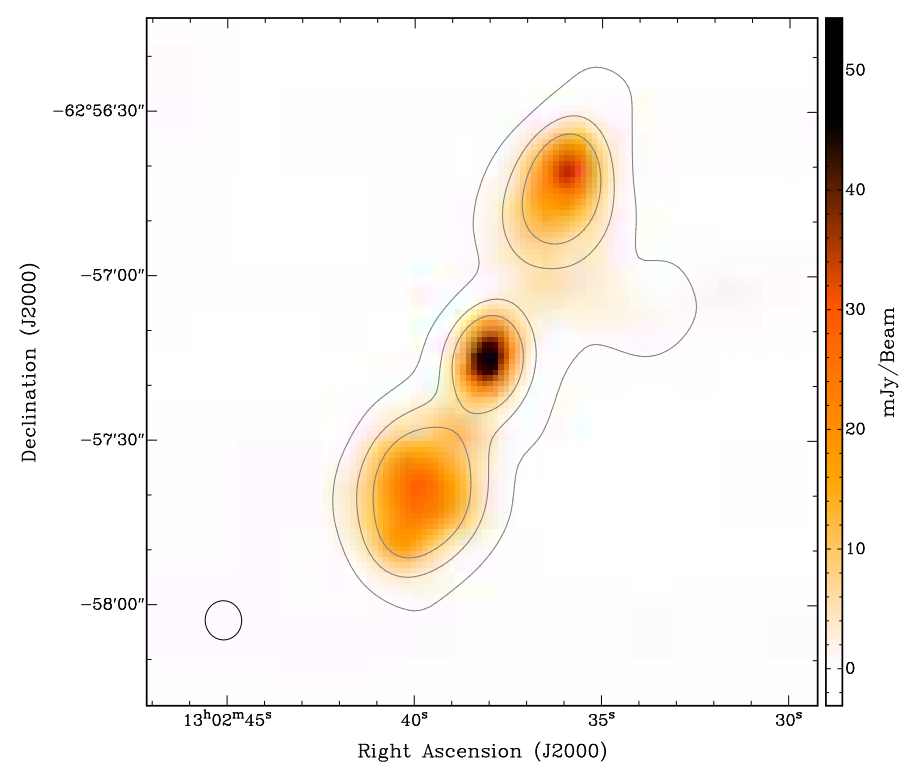

Fig. 3. Radio map of MGPS J130237-625718 at $2.368 \mathrm{GHz}$ overlaid with contours indicating the significance of the emission at $1.384 \mathrm{GHz}$ at the level of 10,40, and 80 times the rms noise. The synthesised beam is shown with a black ellipse in the bottom left corner.

source detected in the PMN survey at $4.85 \mathrm{GHz}$ (Condon et al. 1993; Wright et al. 1994). The emission from PMN J1303-6259 was fitted with a two-dimensional asymmetric Gaussian with major and minor axis widths of $\sigma_{x}=14.28^{\prime}$ and $\sigma_{y}=5.88^{\prime}$ with a position angle of $1.5^{\circ}$ measured eastwards from the north direction (Griffith \& Wright 1993). The total flux density from the source is $235 \pm 14 \mathrm{mJy}$ at $4.85 \mathrm{GHz}$ (Wright et al. 1994). Although PMN J1303-6259 is coincident with the western part of the detected shell-like structure the map also reveals extended radio emission coincident with southern part of the shell (see Fig. 4 middle). Overall, the $4.85 \mathrm{GHz}$ map show a good agreement with the $1.384 \mathrm{GHz}$ morphology of the SNR candidate. To estimate a spectral index of the western part of the SNR candidate, we smoothed the $1.384 \mathrm{GHz}$ map with a Gaussian with a width equal to the angular resolution of the PMN survey of $4.2^{\prime}$ and fitted the region of PMN J1303-6259 assuming the same extension of the source as obtained for PMN J1303-6259 using the miriad task imfit. The obtained flux density at $1.384 \mathrm{GHz}$ is $398 \pm 3 \mathrm{mJy}$ resulting in a spectral index of $\alpha=0.42 \pm 0.05^{1}$, which is in a perfect agreement with a range of values observed for SNRs, i.e. from $\sim 0.2$ to $\sim 0.8$ with a peak at around 0.5 (Reynolds 2011; Green 2014), and very close to a canonical $\alpha=0.5$ expected in the diffusive shock acceleration in the case of strong shocks with a compression ratio of 4 (see e.g. Dubner \& Giacani 2015, and references therein).

Neither the compact source catalogue nor the SNR catalogue of the second epoch Molonglo Galactic Plane Survey (MGPS-2) at $843 \mathrm{MHz}$ (Murphy et al. 2007; Green et al. 2014) provide any counterpart for the SNR candidate. However, the MGPS-2 image of the G304.4-0.2 region at $843 \mathrm{MHz}$ reveals a faint extended radio emission coincident with the SNR candidate, roughly following the $1.384 \mathrm{GHz}$ contours in the west and south regions but also exhibiting emission towards north-east (Fig. 4, right). The peak flux density reaches $17.5 \mathrm{mJy} / \mathrm{beam}$, which is well above the sensitivity of the survey of $2 \mathrm{mJy} / \mathrm{beam}$. The image is strongly distorted by artefacts produced by nearby bright

\footnotetext{
1 Flux density, $S_{v}$, scales with frequency, $v$, as $S_{v} \propto v^{-\alpha}$.
}

sources, which makes it impossible to classify the morphology of the extended emission. This might be the reason why the source did not appear in the list of the SNR candidates detected in the MGPS-2 survey as the search criteria include a condition on a morphology that has to be shell-like or composite (Green et al. 2014).

Infrared and optical observations, for example the Two Micron All-Sky Survey (2MASS) in the $H$-band $(1.65 \mu \mathrm{m}$; Skrutskie et al. 2006), do not show any extended emission from the region of G304.4-0.2. This further suggests that the radio emission from the SNR candidate is most probably non-thermal as expected for SNRs.

Available X-ray observations ${ }^{2}$ of this region show no evidence of the large scale structures coincident with G304.4-0.2. This, however, does not contradict the SNR hypothesis. The absence of the non-thermal X-ray emission can be explained by a potentially old age of the SNR candidate (see below). Indeed, $\mathrm{X}$-ray synchrotron emission from SNRs requires relatively high shock velocities of $\gtrsim 2000 \mathrm{~km} \mathrm{~s}^{-1}$ to accelerate electrons to energies high enough and these shock velocities are believed to be associated with young SNRs of $\sim 1000 \mathrm{yr}$ (see e.g. Vink 2012). Old age might also be a reason for the lack of the X-ray thermal emission. Slowing down of the shock to $<200 \mathrm{~km} \mathrm{~s}^{-1}$ in old SNRs of $\gtrsim 10 \mathrm{kyr}$ results in cooling of the post-shock region to temperatures lower than required for the X-ray emission (see e.g. Vink 2012). Some old SNRs, however, feature thermal $\mathrm{X}$-ray emission from their interior that might be due to, for example, interaction with dense cloudlets, which survive the forward shock crossing and slowly evaporate inside the remnant because of saturated thermal conduction (see e.g. White \& Long 1991; Sushch et al. 2011). Another possible reason for the lack of the thermal emission might be a low density of the ambient medium around the remnant. How low should this density be is, however, model dependent and depends on whether ionization quilibrium is sustained. Non-equilibirum ionization models coupled to efficient particle acceleration shows that X-ray thermal emission would dominate over synchrotron emission only for relatively high densities of about $1 \mathrm{~cm}^{-3}$ (Ellison et al. 2007). The estimate of the density also depends on the distance to the remnant, which is not clear in this case. Finally, the lack of detection of $\mathrm{X}$-ray emission from the source, both thermal and non-thermal, might be due to insufficient sensitivity or exposure.

At $\gamma$-ray energies most of the field of view is covered by HESS J1303-631 and it is impossible to distinguish any emission associated with the possible SNR.

However, other possible interpretations of the detected extended emission cannot be firmly ruled out. Owing to the faint emission, the image of G304.4-0.2 appears to be patchy and this makes it difficult to firmly identify the shell-like morphology. The emission could also be contaminated by unrelated point sources and the image might be distorted by artefacts created by bright sources in the field of view. Since the shell-like morphology of the source is the strongest argument in favour of its SNR nature, we have to treat our conclusions with caution. The observed large scale structure might still be a composition of individual background sources and/or HII regions.

2 All observations of the region of interest available at NASA's HEASARC archive (https://heasarc.gsfc.nasa.gov/docs/ archive.html) were examined including XMM-Newton, Swift, ROSAT, Chandra, and ASCA. 

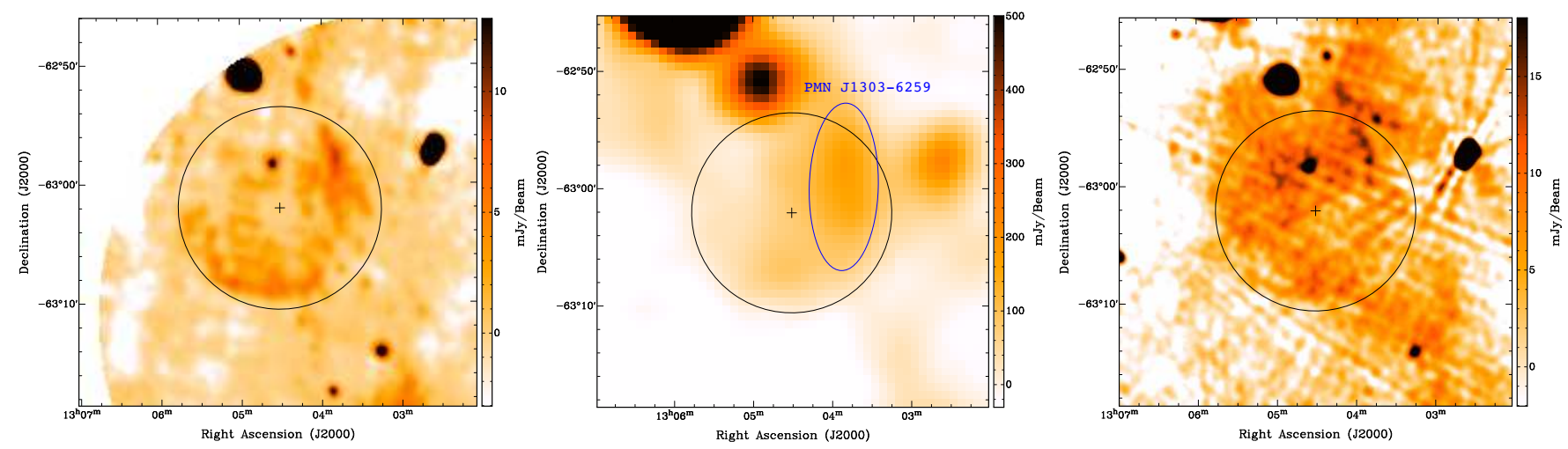

Fig. 4. Left: radio map of the G304.4-0.2 region at $1.384 \mathrm{GHz}$. Middle: radio map of the G304.4-0.2 region at $4.85 \mathrm{GHz}$ obtained in the PMN survey (Condon et al. 1993). The blue ellipse shows the extension of the PMN survey source PMN J1303-6259 as presented in the catalogue (Wright et al. 1994). The resolution of this map is $\sim 5^{\prime}$ (Condon et al. 1993). Right: radio map of the G304.4-0.2 region at $843 \mathrm{MHz}$ obtained in the MGPS-2 survey (Murphy et al. 2007). The size of the beam is $\sim 45^{\prime \prime} \times 50^{\prime \prime}$. The black circle in all the panels indicates the extension of the shell-like structure and the black cross shows its centre. Middle and right images were obtained using the NASA SkyView online tool (http://skyview.gsfc.nasa.gov).

\subsection{Possible birth place of PSRJ1301-6305}

If G304.4-0.2 is indeed an SNR it could be a birth place of the pulsar PSR J1301-6305. This hypothesis is supported by the direction of the X-ray "tail" which roughly points to the position of the SNR candidate (see Fig. 2). The pulsar is located outside the shell-like structure, which means that in case the SNR candidate is indeed the birth place of PSR J1301-6305, the pulsar has already escaped the remnant and continues to propagate in the ambient medium. The larger size of the PWN does not contradict this hypothesis as the present time nebula could have formed after the pulsar escaped the SNR. While the pulsar is still inside the SNR, its nebula is strongly disrupted by the reverse shock of the remnant (see e.g. Blondin et al. 2001; Vorster et al. 2013a) and at the moment of interaction with the shell it is very small. Moving outside the remnant the pulsar builds up a new nebula that can become very large because of the proper motion of the pulsar, i.e. left behind electrons, and the diffusion of electrons in the medium. Escaping the SNR, the pulsar should also damage the shell of the remnant. Although the emission in the direction of the pulsar is slightly fainter, exhibiting a gap in the shell, there is no clear evidence of distortion. This can be naturally explained if the pulsar is not moving in the projected plane but its velocity has a considerable component that is perpendicular to the projected plane. In this case the distorted part of the shell is facing the observer and is thus not visible. The angular distance between the pulsar and the centre of the SNR candidate of about $19^{\prime}$ corresponds to a projected distance of $36 \mathrm{pc}$ assuming the distance to the pulsar of $6.6 \mathrm{kpc}$. This corresponds to a projected pulsar velocity of $V_{\mathrm{p}} \simeq 3100 \mathrm{~km} \mathrm{~s}^{-1}$ for the characteristic age of the pulsar of $11 \mathrm{ky}$ (Manchester et al. 2005). This velocity would make PSR J1301-6305 the fastest known pulsar. The highest pulsar velocity detected so far is $\sim 1600 \mathrm{~km} \mathrm{~s}^{-1}$ (Cordes \& Chernoff 1998). However, there are indications that some pulsars might be much faster with velocities comparable to the estimate presented here for PSR J1301-6305. The estimate of the kick velocity of the possible pulsar IGR J11014-6103 is $2400-2900 \mathrm{~km} \mathrm{~s}^{-1}$ (Tomsick et al. 2012), but the source nature cannot be unambiguously proven yet as no pulsations were detected so far. Also, the real age of PSR J1301-6305 might be higher than the characteristic age if the braking index is lower than 3 , which is the case for eight out of nine pulsars for which the braking index has been reliably measured (Lyne et al. 2015; Archibald et al. 2016). In this case the estimate of the pulsar velocity would be lower.

Alternatively, the real age of the pulsar can be estimated as the age of its SNR. In case G304.4-0.2 is indeed an SNR and the birth place of the pulsar PSR J1301-6305, its size can provide an estimate for the pulsar age. Assuming a distance to the pulsar of $6.6 \mathrm{kpc}$ (Abramowski et al. 2012), the angular size of the SNR candidate of about $16^{\prime}$ corresponds to $30 \mathrm{pc}$ in diameter. The Sedov solution (Sedov 1959), which describes the hydrodynamical expansion of an SNR in the adiabatic stage of evolution into the homogeneous medium, provides an estimate of the SNR age for a given size of the remnant

$t_{\text {age }}=16\left(\frac{E}{10^{51}[\mathrm{erg}]}\right)^{-1 / 2}\left(\frac{n_{\mathrm{ISM}}}{1\left[\mathrm{~cm}^{-3}\right]}\right)^{1 / 2}\left(\frac{R}{15[\mathrm{pc}]}\right)^{5 / 2}[\mathrm{kyr}]$

where $E$ is the explosion energy, $n_{\mathrm{ISM}}$ is the number density of the interstellar medium, and $R$ is the radius of the remnant. This value is somewhat higher than the characteristic age of the pulsar of $11 \mathrm{kyr}$ (Manchester et al. 2005), but does not contradict it if the braking index is lower than 3 (see above). If we assume that the real age of the pulsar is $16 \mathrm{kyr}$ then the projected velocity would be $2100 \mathrm{~km} \mathrm{~s}^{-1}$, which is still very high. The SNR age estimate, however, is dependent on the ambient medium density, which is often considerably lower than $1 \mathrm{~cm}^{-3}$ even by orders of magnitude, and it is also very sensitive to the estimated physical size of the SNR. Therefore, this estimate of age should be taken with caution.

A pulsar with this high velocity moves supersonically in the interstellar medium creating a bow shock. A bow shock driven through the neutral gas can generate optical emission in the Balmer lines at the forward shock (Bucciantini \& Bandiera 2001; Bucciantini 2002) and such emission is already discovered for a few pulsars in $\mathrm{H}_{\alpha}$. There is no evidence for such emission around PSR J1301-6305, which however does not necessarily contradict our hypothesis, since a lot of pulsars are believed to be moving supersonically and bow-shock structures are only detected at optical wavelengths for a few of these objects. It has been noted that $\mathrm{H}_{\alpha}$ bowshocks and X-ray tails are rarely seen together (Kargaltsev et al. 2015). Inside the bowshock pulsar wind particles are accelerated at the termination 
shock subsequently generating non-thermal synchrotron emission that can be detected in radio and X-rays (Gaensler \& Slane 2006). Although no extended radio emission associated with PSR J1301-6305 was detected, which can simply be because of the potentially large size of the radio nebula, the X-ray nebula exhibits a bow-shock morphology with a tail pointing in the direction of G304.4-0.2; this strongly suggests that the pulsar is moving with high velocity.

\section{Summary}

The ATCA observations of the HESS J1303-631 region at $5.5 \mathrm{GHz}$ and $7.5 \mathrm{GHz}$ do not reveal any significant extended emission associated with PSR J1301-6305. Archival $1.384 \mathrm{GHz}$ and $2.368 \mathrm{GHz}$ data also do not show any evidence of a radio counterpart of HESS J1303-631. Non-detection of this evolved PWN at radio wavelengths suggests that either the putative radio PWN is comparable to the size of the GeV counterpart and, thus, larger than the largest reliably imaged structure and even the primary beam of radio observations or that the magnetic field is rather low, which is in agreement with the evolved PWN identification because the magnetic field is expected to decrease with time in PWNe. The comparison of the X-ray emission to the $\mathrm{TeV}$ emission implies the average magnetic field in the PWN of $\sim 0.2-2 \mu \mathrm{G}$ (Abramowski et al. 2012), which however does not exclude the possibility of enhanced magnetic field around the pulsar.

Archival $1.384 \mathrm{GHz}$ observations reveal a detection of an extended structure centred at the angular distance of $19^{\prime}$ from the pulsar. This extended structure might be a SNR and a potential birth place of the pulsar. If this is the case then the projected velocity of the pulsar would be $3100 \mathrm{~km} \mathrm{~s}^{-1}$ assuming the characteristic age of the pulsar. This would make PSR J1301-6305 the fastest known pulsar. However, the uncertainty of the true age of the pulsar can significantly change this estimate.

Acknowledgements. We would like to thank the anonymous referee for valuable comments that strongly improved the paper. We would like to acknowledge the help of Marek Jamrozy (Astronomical Observatory of the Jagiellonian University of Kraków, Poland) and Michael Bietenholz (Hartebeesthoek Radio Observatory, South Africa; York University, Toronto, Canada) on understanding certain aspects of the ATCA analysis performed in the paper. The Australia Telescope Compact Array is part of the Australia Telescope National Facility, which is funded by the Australian Government for operation as a National Facility managed by CSIRO. This paper includes archived data obtained through the Australia Telescope Online Archive (http://atoa.atnf.csiro.au).

\section{References}

Abramowski, A., Acero, F., et al. (H.E.S.S. Collaboration) 2012, A\&A, 548, A46 Acero, F., Ackermann, M., Ajello, M., et al. 2013, ApJ, 773, 77

Aharonian, F., Akhperjanian, A. G., Aye, K.-M., et al. 2005, A\&A, 439, 1013

Archibald, R. F., Gotthelf, E. V., Ferdman, R. D., et al. 2016, ApJ, 819, L16

Benjamin, R. A., Churchwell, E., Babler, B. L., et al. 2003, PASP, 115, 953

Blondin, J. M., Chevalier, R. A., \& Frierson, D. M. 2001, ApJ, 563, 806

Bucciantini, N. 2002, A\&A, 393, 629

Bucciantini, N., \& Bandiera, R. 2001, A\&A, 375, 1032

Condon, J. J., Griffith, M. R., \& Wright, A. E. 1993, AJ, 106, 1095

Cordes, J. M., \& Chernoff, D. F. 1998, ApJ, 505, 315

Cutri, R. M., Skrutskie, M. F., van Dyk, S., et al. 2003, VizieR Online Data Catalog: II/246

de Jager, O. C., Ferreira, S. E. S., Djannati-Ataï, A., et al. 2009, ArXiv e-prints [arXiv: 0906.2644]

Dubner, G., \& Giacani, E. 2015, A\&ARv, 23, 3

Ellison, D. C., Patnaude, D. J., Slane, P., Blasi, P., \& Gabici, S. 2007, ApJ, 661, 879

Gaensler, B. M., \& Slane, P. O. 2006, ARA\&A, 44, 17

Gelfand, J. D., Castro, D., Slane, P. O., et al. 2013, ApJ, 777, 148

Gooch, R. 1995, in Astronomical Data Analysis Software and Systems IV, eds.

R. A. Shaw, H. E. Payne, \& J. J. E. Hayes, ASP Conf. Ser., 77, 144

Green, D. A. 2014, Bull. Astron. Soc. India, 42, 47

Green, A. J., Reeves, S. N., \& Murphy, T. 2014, PASA, 31, e042

Griffith, M. R., \& Wright, A. E. 1993, AJ, 105, 1666

Kargaltsev, O., Cerutti, B., Lyubarsky, Y., \& Striani, E. 2015, Space Sci. Rev., 191,391

Lyne, A. G., Jordan, C. A., Graham-Smith, F., et al. 2015, MNRAS, 446, 857

Manchester, R. N., Hobbs, G. B., Teoh, A., \& Hobbs, M. 2005, AJ, 129, 1993

Misanovic, Z., Cram, L., \& Green, A. 2002, MNRAS, 335, 114

Monet, D. G., Levine, S. E., Canzian, B., et al. 2003, AJ, 125, 984

Murphy, T., Mauch, T., Green, A., et al. 2007, MNRAS, 382, 382

Porth, O., Komissarov, S. S., \& Keppens, R. 2014, MNRAS, 438, 278

Reynolds, S. P. 2011, Ap\&SS, 336, 257

Rosen, S. R., Webb, N. A., Watson, M. G., et al. 2016, A\&A, 590, A1

Sault, R. J., Teuben, P. J., \& Wright, M. C. H. 1995, in Astronomical Data Analysis Software and Systems IV, eds. R. A. Shaw, H. E. Payne, \& J. J. E. Hayes, ASP Conf. Ser., 77, 433

Schinzel, F. K., Petrov, L., Taylor, G. B., et al. 2015, ApJS, 217, 4

Sedov, L. I. 1959, Similarity and Dimensional Methods in Mechanics (New York: Academic Press)

Simpson, R. J., Povich, M. S., Kendrew, S., et al. 2012, MNRAS, 424, 2442

Skrutskie, M. F., Cutri, R. M., Stiening, R., et al. 2006, AJ, 131, 1163

Sushch, I., Hnatyk, B., \& Neronov, A. 2011, A\&A, 525, A154

Tomsick, J. A., Bodaghee, A., Rodriguez, J., et al. 2012, ApJ, 750, L39

Vink, J. 2012, A\&ARv, 20, 49

Vorster, M. J., Ferreira, S. E. S., de Jager, O. C., \& Djannati-Ataï, A. 2013a, A\&A, 551, A127

Vorster, M. J., Tibolla, O., Ferreira, S. E. S., \& Kaufmann, S. 2013b, ApJ, 773, 139

White, R. L., \& Long, K. S. 1991, ApJ, 373, 543

Wright, A. E., Griffith, M. R., Burke, B. F., \& Ekers, R. D. 1994, ApJS, 91, 111

Wright, E. L., Eisenhardt, P. R. M., Mainzer, A. K., et al. 2010, AJ, 140, 1868

Wu, J. H. K., Wu, E. M. H., Hui, C. Y., et al. 2011, ApJ, 740, L12 